\title{
Effects of Pre-Schooler Lifestyle on the Circadian Rhythm of Secretory Immunoglobulin A
}

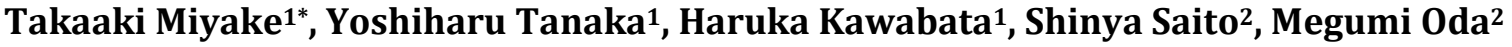 \\ ${ }^{1}$ Faculty of Liberal Arts and Sciences, Osaka Prefecture University, Osaka, Japan \\ ${ }^{2}$ Graduate School of Health Sciences, Okayama University, Okayama, Japan \\ Email: *tmiyake@las.osakafu-u.ac.jp
}

How to cite this paper: Miyake, T., Tanaka, Y., Kawabata, H., Saito, S. and Oda, M. (2021) Effects of Pre-Schooler Lifestyle on the Circadian Rhythm of Secretory Immunoglobulin A. Health, 13, 178-187. https://doi.org/10.4236/health.2021.132016

Received: January 18, 2021

Accepted: February 23, 2021

Published: February 26, 2021

Copyright (c) 2021 by author(s) and Scientific Research Publishing Inc. This work is licensed under the Creative Commons Attribution International License (CC BY 4.0).

http://creativecommons.org/licenses/by/4.0/

\begin{abstract}
Assuming that secretory immunoglobulin A (s-IgA) in pre-schoolers is influenced by wake-up time, bedtime, sleeping duration and activity, the current study examined the influence of basic pre-schooler lifestyle on the circadian rhythm of s-IgA. We conducted a questionnaire survey on lifestyle and measured steps, heart rate and salivary s-IgA in 44 pre-schoolers aged 4 or 5 years old. Saliva was collected over 2 days at six time points: bedtime, upon waking up, upon arriving at nursery school, before lunch, after lunch and upon going home. To obtain relatively reliable s-IgA values, the $s-\operatorname{IgA}$ concentration/total protein concentration ratio (referred to as s-IgA/T.P.) was calculated. Relationship between the s-IgA/T.P. and heart rate, steps, wake-up time, bedtime and sleeping duration was then examined using Pearson's correlation coefficient. Accordingly, pre-schooler lifestyle had the following effect on circadian rhythm of s-IgA: 1) s-IgA/T.P. values were higher upon waking up than at other measurement time points; 2) pre-schoolers with considerable physical activity had high s-IgA/T.P. at bedtime and upon waking up; and 3) pre-schoolers with long sleeping durations and early bedtime had high s-IgA/T.P. upon waking up. Thus, our results confirmed that pre-schoolers' lifestyle habits, such as bedtime, sleeping duration, physical activity, had an effect on the circadian rhythm of s-IgA.
\end{abstract}

\section{Keywords}

Pre-Schooler, s-IgA, Lifestyle, Activity, Sleep

\section{Introduction}

Establishing an appropriate lifestyle during early childhood is imperative for 
creating a healthy life thereafter. As such, the Ministry of Education, Culture, Sports, Science and Technology of Japan (MEXT) has been actively working to establish correct lifestyles for children and improve their lifestyle rhythms by promoting the national movement of "early sleep, get up early and breakfast" since 2006. So far, most research on pre-schooler healthy has involved surveys conducted using questionnaires, with only a handful utilizing biochemical indicators. To better determining the effects of pre-schooler lifestyle, understanding how it affects the pre-schooler's physiology utilizing objective biochemical indicators is necessary.

Recently, non-invasive methods, such as saliva collection, have made measuring secretory-immunoglobulin A (s-IgA) convenient. s-IgA contained in exocrine products, such as saliva, digestive juices, airway secretions and breast milk, is an immune protein that plays an essential role in host defence and local immune system function at the mucosal epithelium of the digestive and respiratory systems [1] [2]. s-IgA is largely found in the saliva and early breast milk and attacks invading bacteria and viruses present on the mucous membranes of the oral and upper respiratory tract [3] [4]. Therefore, measuring salivary s-IgA levels in pre-schoolers can help us determine the local immunologic capability of their oral and nasal cavities, which are important for defence against upper respiratory tract infection.

Given that most studies concerning s-IgA have been on adults, research on s-IgA in pre-schoolers has been scarce. Ichikawa [5] who analysed salivary s-IgA upon waking up in 330 sixth graders, 152 junior high school sophomores and 334 adults (over 20 years) reported no gender difference in salivary s-IgA among children and increased levels with aging. Moreover, they found that adults who were long-term smokers and patients with bronchial asthma showed significantly lower s-IgA concentrations compared to the general population. Maeda [6] revealed that patients with bronchial asthma aged 5 - 15 years had lower salivary s-IgA levels than healthy children, indicating lowered local immunologic ability. In addition, Sakai et al. [2] reported no gender difference in salivary s-IgA levels in both children and adults; lower s-IgA in children than in adults; and large variations in s-IgA among adults depending on circadian rhythm. Suzukawa et al. [7], who examined the relationship between lifestyle and s-IgA in pre-schoolers, reported that pre-schoolers with little exercise play and those who slept for less than $9 \mathrm{~h}$ showed lower s-IgA values, with no gender difference. After examining s-IgA variations at 10:30 am, 3:30 pm and 8:00 pm on weekdays and weekends in pre-schoolers aged 4 or 5 years old, Watamura et al. [8] reported that the salivary s-IgA levels were higher at 10:30 am than s-IgA levels at 8:00 pm on weekdays and weekends. Lim et al. [9] analysed s-IgA in saliva twice at $7-9$ am and $7-9 \mathrm{pm}$ and reported that the salivary s-IgA was significantly higher in morning than evening regardless of age, gender and race. Thus, given the influence of age, bronchial asthma, exercise habits and sleep duration on s-IgA levels in pre-schoolers, determining the influence of basic lifestyle patterns on pre-schoolers' s-IgA is necessary. 
The current study examined the influence of basic pre-schooler lifestyle on the circadian rhythm of s-IgA with the assumption that pre-schooler s-IgA levels are influenced by wake-up time, bedtime, sleeping duration and activity.

\section{Methods}

\subsection{Subjects}

Subjects were pre-schoolers aged 4 or 5 years old, belonging to K Nursery School of Tokyo prefecture and Y Nursery School of Yamaguchi prefecture. They were included in this study because they had similar living backgrounds. Parents of the pre-schoolers were provided a document detailing the aims of study. Among the 64 pre-schoolers who provided informed consent, 44 who correctly answered the questionnaire and permitted saliva measurements were selected for this study.

\subsection{Questionnaire and Measurement Content}

\subsubsection{Lifestyle Questionnaire}

Parents provided informed consent underwent a questionnaire survey on lifestyle of pre-schoolers. The survey form stated that consideration would be given to the protection of personal information. Class teachers at the nursery schools were requested to distribute and collect the questionnaires. This survey was conducted in 2007.

\subsubsection{Measuring Heart Rate and Amount of Activity}

To understand daytime activity levels of pre-schoolers, steps and heart rates were measured in 21 pre-schoolers whose parents provided consent. Daily steps during childcare, which indicated the amount of physical activity, was measured by fixing the Calorie Counter Acceleration Pedometer (SUZUKEN Co. Ltd.) to the waist of pre-schoolers. Heart rates (heart rate per unit time) during childcare were measured using a Portable Cardiograph (VINE Co. Ltd.) with the chest bipolar induction method.

\subsubsection{Measurement of Secretory Immunoglobulin $A$}

Saliva was collected during the 2 days of routine childcare at the following six time points: bedtime, upon waking up, upon arriving at the nursery school (9:00-9:30 am), before lunch (11:00 am), after lunch (13:00 pm) and upon going home. On the day of saliva collection, parents were asked regarding the following condition of the pre-schoolers: "No pharmacological therapy," "no special exercise and attention needed to live normally," "consideration given to normalize sleep rhythm and eating rhythm," "no dental treatment," and "consideration given to prevent mental stressors." Approximately $1-2 \mathrm{~mL}$ of saliva directly from the mouth was collected into a $50-\mathrm{mL}$ centrifuge tube given that this was the easiest method of collecting saliva from pre-schoolers. Immediately, the saliva was transferred into a $1.5-\mathrm{mL}$ microtube and frozen at $-20^{\circ} \mathrm{C}$. Saliva samples were analysed for s-IgA levels after centrifugation for $5 \mathrm{~min}$ at $4^{\circ} \mathrm{C}$ and 15,000 
rpm and transferring the supernatant into a new tube.

\subsubsection{Saliva Analysis Method}

s-IgA concentrations were measured using Enzyme-Linked ImmunoSorbent Assay (ELISA). s-IgA concentrations in saliva have been known to fluctuate depending on total protein secretion, collection time, collection method and treatment method [2]. Hence, to obtain a relatively reliable s-IgA value, total protein measurements in saliva were performed using the Lowry method, then the s-IgA value was calculated as the s-IgA concentration/total protein concentration ratio (referred to as s-IgA/T.P.).

\subsection{Statistical Analysis}

The z-test was used to compare wake-up time, bedtime and sleeping duration obtained from this study and those obtained from the survey of Hattori et al. [10], as well as a health survey on wake-up time and bedtime according to measurement time zone [11]. Differences in average s-IgA/T.P. according to measurement time points were examined using one-way analysis of variance. The relationship between s-IgA/T.P. and heart rate, steps, wake-up time, bedtime and sleeping duration was examined using Pearson's correlation coefficient, with the level of significance set at 0.05 .

\subsection{Ethical Considerations}

The Ethics Committee on Human Experimentation of the Graduate School of Health Sciences, Okayama University approved this study (Approval No. D10-03).

\section{Results}

In order to confirm that the subjects of this study have standard lifestyles, we compared them with a previous study by Hattori et al. [10] and a health survey on pre-schoolers [11], which included large number of subjects. Table 1 shows the average wake-up time, bedtime and sleeping duration of subjects included herein and those included in a previous study by Hattori et al. [10]. Accordingly, pre-schoolers had an average wake-up time of 7:06 $\pm 30 \mathrm{~min}$, average bedtime of $21: 36 \pm 41 \mathrm{~min}$ and average sleep duration (calculated from the wake-up time and bedtime) of $9.5 \mathrm{~h} \pm 36 \mathrm{~min}$. The results of this study were not significantly

Table 1. Comparison of average wake-up time, bedtime and sleep duration between this study and Hattori's study on pre-schoolers.

\begin{tabular}{|c|c|c|c|c|c|c|}
\hline & \multicolumn{2}{|c|}{$\begin{array}{c}\text { this study on } \\
\text { pre-schoolers } \mathrm{N}=44\end{array}$} & \multicolumn{2}{|c|}{$\begin{array}{c}\text { Hattori's study on } \\
\text { pre-schoolers } N=459\end{array}$} & \multirow[t]{2}{*}{$\mathbf{z}$} & \multirow{2}{*}{$\mathrm{p}$} \\
\hline & M & SD & M & SD & & \\
\hline wake-up time & $7: 06$ & $30 \min$ & $7: 07$ & $25 \mathrm{~min}$ & -1.579 & 0.114 \\
\hline bedtime & $21: 36$ & $41 \mathrm{~min}$ & $21: 17$ & $38 \mathrm{~min}$ & 0.316 & 0.752 \\
\hline sleeping duration & 9.5 hour & $36 \mathrm{~min}$ & 9.8 hour & $45 \mathrm{~min}$ & 1.769 & 0.077 \\
\hline
\end{tabular}


different from the survey by Hattori et al. [10].

Table 2 and Table 3 show distribution of wake-up time and bedtime, respectively, according to each measurement time zone in this study and a health survey on pre-schoolers [11]. A wake-up time of 7 - 8 am and bedtime of 9 - 10 pm were the most frequently observed, with no significant difference between this study and the health survey on pre-schoolers.

The average heart rate of 21 pre-schoolers during childcare was $114.9 \pm 10.9$ beats/min, with maximum and minimum values of 199 and 70 beats/min, respectively. The average step count was $7405 \pm 2923$, with maximum and minimum values of 12,031 and 3100, respectively.

Circadian rhythm of s-IgA/T.P. from bedtime until leaving nursery school the following next day is shown in Figure 1. The average s-IgA/T.P. was $10.6 \% \pm$

Table 2. Distribution of wake-up time according to each measurement time zone in this study and the health survey on pre-schoolers (2011).

\begin{tabular}{ccccc}
\hline & $\begin{array}{c}\text { this study on } \\
\text { pre-schoolers } \mathbf{N}=\mathbf{4 4}(\%)\end{array}$ & $\begin{array}{c}\text { health survey on } \\
\text { pre-schoolers } \mathbf{N}=\mathbf{9 3 1}(\%)\end{array}$ & $\mathbf{z}$ & $\mathbf{p}$ \\
\hline before $7 \mathrm{am}$ & $14(31.8)$ & $232(24.9)$ & 1.030 & 0.303 \\
7 - $8 \mathrm{am}$ & $25(56.8)$ & $580(62.3)$ & -0.732 & 0.464 \\
after $8 \mathrm{am}$ & $5(11.4)$ & $102(11.0)$ & 0.085 & 0.933 \\
N.A. & $0(0.0)$ & $17(1.8)$ & -0.898 & 0.369 \\
\hline
\end{tabular}

Table 3. Distribution of bedtime according to each measurement time zone in this study and the health survey on pre-schoolers (2011).

\begin{tabular}{ccccc}
\hline & $\begin{array}{c}\text { this study on } \\
\text { pre-schoolers } \mathbf{N}=\mathbf{4 4}(\%)\end{array}$ & $\begin{array}{c}\text { health survey on } \\
\text { pre-schoolers } \mathbf{N}=\mathbf{9 3 1}(\%)\end{array}$ & $\mathbf{z}$ & $\mathrm{p}$ \\
\hline before $9 \mathrm{pm}$ & $2(4.5)$ & $120(12.9)$ & -1.635 & 0.102 \\
$9-10 \mathrm{pm}$ & $29(65.9)$ & $533(57.3)$ & 1.136 & 0.256 \\
after $10 \mathrm{pm}$ & $13(29.6)$ & $233(25.0)$ & 0.674 & 0.500 \\
N.A. & $0(0.0)$ & $45(4.8)$ & -1.488 & 0.137 \\
\hline
\end{tabular}

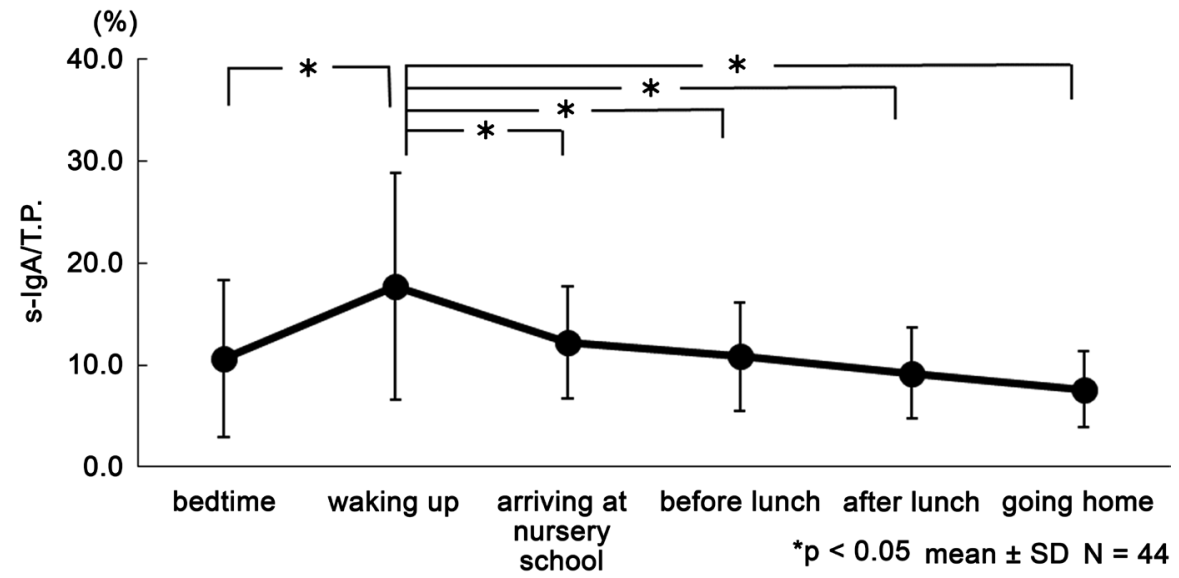

Figure 1. Circadian rhythm of s-IgA/T.P. 
Table 4. Correlation coefficient between s-IgA/T.P. at each measurement time point and heart rate, steps, wake-up time, bedtime and sleeping duration.

\begin{tabular}{ccccccc}
\hline & bedtime & waking up & $\begin{array}{c}\text { arriving at } \\
\text { nursery school }\end{array}$ & $\begin{array}{c}\text { before } \\
\text { lunch }\end{array}$ & after lunch & $\begin{array}{c}\text { going } \\
\text { home }\end{array}$ \\
\hline heart rate & 0.032 & 0.045 & $-0.444^{*}$ & -0.408 & -0.241 & -0.277 \\
steps & $0.717^{*}$ & $0.552^{*}$ & -0.008 & 0.120 & 0.316 & 0.328 \\
wake-up time & -0.091 & -0.178 & 0.211 & 0.021 & -0.157 & -0.094 \\
bedtime & -0.291 & $-0.427^{*}$ & 0.048 & -0.109 & $-0.309^{*}$ & -0.167 \\
sleeping duration & 0.173 & $0.337^{*}$ & 0.123 & 0.142 & 0.221 & 0.111 \\
\hline${ }^{*} \mathrm{p}<0.05$. & & & & & &
\end{tabular}

7.7\% before bedtime, $17.7 \% \pm 11.1 \%$ upon waking up, $12.2 \% \pm 5.5 \%$ upon arrive at nursery school, $10.8 \% \pm 5.3 \%$ before lunch, $9.2 \% \pm 4.5 \%$ after lunch and $7.6 \%$ $\pm 3.8 \%$ upon going home. The s-IgA/T.P. ratio upon waking up was found to be significantly higher than that during other measurement time points.

Table 4 shows the correlation coefficient between s-IgA/T.P. at each saliva collection time and heart rate, steps, wake-up time, bedtime and sleeping duration. A significant negative correlation was found between heart rate and s-IgA/T.P. upon arriving at nursery school, while a significant positive correlation was found between steps and s-IgA/T.P. at bedtime and upon waking up. A significant negative correlation was found between bedtime and s-IgA/T.P. upon waking up and after lunch, while a significant positive correlation was found between sleeping duration and s-IgA/T.P. upon waking up.

\section{Discussion}

The average wake-up time, bedtime and sleep duration obtained herein did not differ from those obtained by Hattori et al. [10]. Moreover, no difference in wake-up time and bedtime was observed according to measurement time zone between this study and the health survey on pre-schoolers [11]. Hence, the current study had been confirmed to have included 4 - 5-year-old pre-schoolers who have sleep and awakening rhythms similar to modern standard 4 - 5-year-old pre-schoolers. However, total sleeping hours for 3 - 5-year-old pre-schoolers recommended by National Sleep Foundation in 2015 remain at $10-13 \mathrm{~h}$ [12]. An international survey by Mindell et al. [13], and Kohyama et al. [14] indicated that Japanese 3-year-old pre-schoolers had less sleep duration compared to those in other countries. Moreover, a cohort study that followed 500 Swiss children for over 16 years showed that 5-year-old pre-schoolers went to be at 20:11 pm bedtime and had $11.1 \mathrm{~h}$ of sleeping time [15]. Thus, the current study, as well as other studies, have indicated that Japanese pre-schoolers had a later bedtime and shorter sleep duration compared to the standard of other countries. In the current study, $29.6 \%$ of 4 or 5 -year-old pre-schoolers went to bed after $10 \mathrm{pm}$, while $9.1 \%$ went to bed after 11:00 pm. In other words, approximately $30 \%$ of pre-schoolers went to bed very late. Mitsuboshi et al. [16] suggested a significant 
relationship between parents' and pre-schoolers' bedtime, thereby indicating that the parent's lifestyle affected the pre-schoolers' bedtime. They reported that $62.9 \%$ of parents were not concerned with their pre-schoolers having a sleep duration of less than $8 \mathrm{~h}$, suggesting a problem with parents' awareness. Considering the potential effects of parent's lifestyle on pre-schoolers, future studies should also examine the impact of parent's lifestyle.

The average heart rate of pre-schoolers during childcare activities obtained herein was 114.9, less than 129 beats/min, was indicated low exercise intensity according to Kobayashi et al. [17]. Moreover, pre-schoolers included herein took an average of 7405 steps, a figure similar to that obtained in previous studies [18] [19] [20].

The s-IgA/T.P. upon waking up was significantly higher than that at other measurement time points from bedtime until going home from nursery school the next day. Nakata et al. [21], who analysed salivary s-IgA five times every $2 \mathrm{~h}$ from 9:00 am to 17:00 pm in adults, showed that s-IgA was highest at 9:00 am, after which a decrease was observed. Rapson et al. [22], who examined s-IgA concentrations $30 \mathrm{~min}, 3 \mathrm{~h}, 6 \mathrm{~h}, 9 \mathrm{~h}$ and $14 \mathrm{~h}$ after waking up, showed that s-IgA upon waking up was the highest, after which a decrease was noted. Moreover, several reports have shown higher s-IgA concentrations upon waking up or in the morning compared to that measured at other time points [23] [24] [25] [26] [27]. Hence, the current study supports previous researches indicating that the decreased s-IgA/T.P. during awakening could be recovered during sleep.

After analysing the correlation coefficient between s-IgA/T.P. at each saliva collection time point and heart rate and steps, a significant negative correlation coefficient was found between heart rate and s-IgA/T.P. upon arriving at nursery school, while a significant correlation was found between steps and s-IgA/T.P. at bedtime and upon waking up. In other words, s-IgA/T.P. decreased when heart rate was high and increased when the amount of steps was high. Adult studies have indeed reported a decrease in s-IgA after vigorous exercise [28] [29] [30]. Suzukawa et al. [7] reported that pre-schoolers who had little exercise and body movement showed low s-IgA values. Thus, examining activity intensity and time management during nursery care of pre-schoolers will be necessary. After analysing the correlation coefficient between salivary s-IgA/T.P. and wake-up time, bedtime and sleep duration at each collection time, the current study found a significant negative correlation between bedtime and s-IgA/T.P. upon waking up and a significant positive correlation between sleeping duration and s-IgA/T.P. upon waking up. In other words, it was clear that pre-schoolers who had a habit of an early bedtime and long sleeping hours had higher s-IgA/T.P. upon waking up, indicating good local immunologic capability in the oral cavity. Suzukawa et al. [7] reported that 4 - 6-year-old pre-schoolers who sleep less $9 \mathrm{~h}$ and hardly had any physical activity had low s-IgA. Moreover, pre-schoolers with habit of long sleeping hours were reported to have significantly more daily steps than those with short sleeping hours [31] [32]. Our results showed that 
long sleep durations and increased physical activity increased s-IgA/T.P. This suggests that increased physical activity following longer sleeping duration improves immune system activity by increasing s-IgA/T.P. Hence, pre-schoolers who have longer sleep durations, early bedtime and engage in physical activity could be less likely affected by diseases, such as catch colds, given that their immune system functions appropriately.

Thus, lifestyle habits, such as bedtime, sleep duration, physical activity were confirmed to have affected the circadian rhythm of s-IgA. Since this survey includes 2 nursery schools and 44 subjects, and only s-IgA is used as an index, it is necessary to analyse more cases and add physiological indicators in order to have universal applicability.

\section{Conclusion}

The current study showed the following effects of pre-schooler lifestyle on the circadian rhythm of s-IgA: 1) s-IgA/T.P. of pre-schoolers was higher upon waking up than that at other measurement time points; 2) pre-schoolers with considerable physical activity had high s-IgA/T.P. at bedtime and upon waking up; and 3) pre-schoolers with long sleep durations and early bedtime had high s-IgA/T.P. upon waking up.

\section{Acknowledgements}

We are grateful to the pre-schoolers and nursery teachers of $\mathrm{Y}$ and $\mathrm{K}$ nursery schools for their cooperation in this study. We are grateful to Professor Yoshimasa Matsuura of Osaka Prefecture University for his advice on this study.

This work was supported by JSPS KAKENHI Grant Number JP19500581.

\section{Conflicts of Interest}

The authors declare no conflicts of interest regarding the publication of this paper.

\section{References}

[1] Evans, P., Hucklebridge, F., Clow, A. and Doyle, A. (1995) Secretory Immunoglobulin A as Convenient Biomarker in Health Survey Work. In: Rodriguez-Martin, J., Ed., Health Psychology and Quality of Life Research, University of Alicante Press, Alicante, 541-549.

[2] Sakai, K., Yamada, H., Takasuka, S., Nakajima, I. and Akasaka, M. (1996) The Study on the Secretory IgA of Human Saliva by the Enzyme Immunoassay: Investigation of S-IgA Concentration, S-IgA/total Protein Ratio between Children and Adults. The Japanese Journal of Pedodontics, 24, 483-494. (in Japanese)

[3] Jemmott III, J.B. and McClelland, D.C. (1989) Secretory IgA as a Measure of Resistance to Infectious Disease: Comments on Stone, Cox, Valdimarsdottir, and Neale. Behavioral Medicine, 15, 63-71. https://doi.org/10.1080/08964289.1989.9935153

[4] Woof, J.M. and Kerr, M.A. (2006) The Function of Immunoglobulin A in Immuni- 
ty. The Journal of Pathology, 208, 270-282. https://doi.org/10.1002/path.1877

[5] Ichikawa, S. (1982) Epidemiological Study on the Estimation of the Effects of Smoking and Respiratory Symptoms and Diseases Using Values of Salivary Albumin, Ig A, and Ig G. Journal of Yokohama Medical Association, 33, 63-79. (in Japanese)

[6] Maeda, M. (1985) Secretory IgA in Immunological Diseases of Infants and Children: Part II. Salivary s-IgA Levels in Patients with Bronchial Asthma. Japanese journal of Allergology, 34, 122-127. (in Japanese)

[7] Suzukawa, K., Nishiyama, T., Ochi, E., Umeda, T., Itoh, T. and Nakamichi, S. (2009) Effects of Exercise and Lifestyle on Immunity in Young Children: Study from SIgA in Saliva. Journal of Research Institute for Sport Science, Nippon Sport Science University, 34, 81-85. (in Japanese)

[8] Watamura, S.E., Coe, C.L., Laudenslager, M.L. and Robertson, S.S. (2010) Child Care Setting Affects Salivary Cortisol and Antibody Secretion in Young Children. Psychoneuroendocrinology, 35, 1156-1166. https://doi.org/10.1016/j.psyneuen.2010.02.001

[9] Lim, P.W., Nambiar, S., Muhardi, L., Abdul Kader, U.H., Garssen, J. and Sandalova, E. (2019) Young Children Display Diurnal Patterns of Salivary IgA and Alpha-Amylase Expression Which Are Independent of Food Intake and Demographic Factors. Biomed Research International, 2019, Article ID: 3687416. https://doi.org/10.1155/2019/3687416

[10] Hattori, S., Adachi, T., Shimazaki, H. and Miyake, T. (2004) The Influence of Television Viewing Time on Infantile Lifestyle. The Journal of Child Health, 63, 516-523. (in Japanese)

[11] The Japanese Society of Child Health (2011) 2010 Infant Health Survey Report. Japanese Society of Child Health, 39. (in Japanese)

[12] Hirshkowitz, M., Whiton, K., Albert, S.M., Alessi, C., Bruni, O., DonCarlos, L., et al. (2015) National Sleep Foundation's Sleep Time Duration Recommendations: Methodology and Results Summary. Sleep Health, 1, 40-43.

https://doi.org/10.1016/j.sleh.2014.12.010

[13] Mindell, J.A., Sadeh, A., Wiegand, B., Hwei, T.H. and Goh, D.Y.T. (2010) Cross-Cultural Differences in Infant and Toddler Sleep. Sleep Medicine, 11, 274-280.

https://doi.org/10.1016/j.sleep.2009.04.012

[14] Kohyama, J., Mindell, J.A. and Sadeh, A. (2011) Sleep Characteristics of Young Children in Japan: Internet Study and Comparison with Other Asian Countries. Pediatrics International, 53, 649-655. https://doi.org/10.1111/j.1442-200X.2010.03318.x

[15] Iglowstein, I., Jenni, O.G., Molinari, L. and Largo, R.H. (2003) Sleep Duration from Infancy to Adolescence: Reference Values and Generational Trends. Pediatrics, 111, 302-307. https://doi.org/10.1542/peds.111.2.302

[16] Mitsuboshi, T., Kato, K., Shimizu, S., Matsumoto, S., Ganno, Y., Inoue, E., et al. (2012) The Factor Which Have an Influence on a Sleep Habits and the Sleep of Japanese Infants. The Journal of Child Health, 71, 808-816. (in Japanese)

[17] Kobayashi, K., Wakita, H. and Yagi, N. (1990) Infant Developmental Kinematics. Minerva Shobo, Kyoto, 194-195. (in Japanese)

[18] Miyake, T., Matsuura, Y. and Shimizu, N. (2004) Effects of Physical Activities on the Body Temperature of Preschoolers. The Journal of Movement and Health Education, 13, 19-26. (in Japanese) 
[19] Nakano, T., Kasuga, K. and Murase, T. (2010) An Examination of the Appropriate Physical Activity Level Related to Lifestyle and Motor Ability for Kindergarten Children. Japan Journal of Human Growth and Development Research, 46, 49-58. (in Japanese) https://doi.org/10.5332/hatsuhatsu.2010.46 49

[20] Miyake, T. (2014) Research on the Actual State and Relationship among the Wake-Up Time and Bedtime, the Amount of Physical Activity and Body Temperature of Young Children. The Journal of Health Education of Children, 20, 39-48. (in Japanese)

[21] Nakata, Y., Iijima, S., Maruyama, S., Tajima, F., Matsuda, T. and Osada, H. (2000) Levels of Salivary Secretory Immunoglobulin A as a Workload Indicator. Japan Air Self-Defense Force Aeromedical Laboratory Reports, 40, 27-35. (in Japanese)

[22] Rapson, A., Collman, E., Faustini, S., Yonel, Z., Chapple, I.L., Drayson, M.T., et al. (2020) Free Light Chains as an Emerging Biomarker in Saliva: Biological Variability and Comparisons with Salivary IgA and Steroid Hormones. Brain, Behavior, and Immunity, 83, 78-86. https://doi.org/10.1016/j.bbi.2019.09.018

[23] Shinada, K., Kawaguchi, Y. and Shimura, N. (1991) A Study of Salivary Immunoglobulin A. I. Daily Change of IgA and Relationship between Salivary IgA and Egogram. Japanese Journal of Psychosomatic Dentistry, 6, 123-128. (in Japanese)

[24] Hucklebridge, F., Clow, A. and Evans, P. (1998) The Relationship between Salivary Secretory Immunoglobulin A and Cortisol: Neuroendocrine Response to Awakening and the Diurnal Cycle. International Journal of Psychophysiology, 31, 69-76. https://doi.org/10.1016/S0167-8760(98)00042-7

[25] Shirakawa, T., Mitome, M. and Oguchi, H. (2004) Circadian Rhythms of S-IgA and Cortisol in Whole Saliva-Compensatory Mechanism of Oral Immune System for Nocturnal Fall of Saliva Secretion-. Pediatric Dental Journal, 14, 115-120. https://doi.org/10.1016/S0917-2394(04)70017-8

[26] Nagamatsu, T. and Kai, Y. (2014) Effect of Low-Intensity Stretching Exercises on Sleep and Stress in People with Mild Sleep Disorders. Bulletin of the Physical Fitness Research Institute, 112, 1-7. (in Japanese)

[27] Kobayashi, H., Song, C., Ikei, H., Park, B.J., Kagawa, T. and Miyazaki, Y. (2017) Diurnal Changes in Distribution Characteristics of Salivary Cortisol and Immunoglobulin A Concentrations. International Journal of Environmental Research and Public Health, 14, 987. https://doi.org/10.3390/ijerph14090987

[28] Tharp, G.D. and Barnes, M.W. (1990) Reduction of Saliva Immunoglobulin Levels by Swim Training. European Journal of Applied and Occupational Physiology, 60, 61-64. https://doi.org/10.1007/BF00572187

[29] MacKinnon, L.T. and Jenkins, D.G. (1993) Decreased Salivary Immunoglobulins after Intense Interval Exercise before and after Training. Medicine \& Science in Sports \& Exercise, 25, 678-683. https://doi.org/10.1249/00005768-199306000-00005

[30] Nieman, D.C., Henson, D.A., Fagoaga, O.R., Utter, A.C., Vinci, D.M., Davis, J.M., et al. (2002) Change in Salivary IgA Following a Competitive Marathon Race. International Journal of Sports Medicine, 23, 69-75. https://doi.org/10.1055/s-2002-19375

[31] Henmi, H., Hagi, Y., Suzuki, S., Ishida, Y., Yamamoto, N. and Yoshitake, Y. (2007) Relationship between the Sleeping Hours and Physical Activity in Preschool Children. Annals of Fitness and Sports Sciences, 35, 15-21. (in Japanese)

[32] Tanaka, S. and Nanakida, A. (2008) A Study on the Correlation of Physical Activity and Life Rhythm among Pre-Schoolers; Based on Results Measured by Accelerometer. Japan Journal of Human Growth and Development Research, 40, 1-10. (in Japanese) 